\title{
Reef Fish in the Mudflats of Kaledupa Island in Wakatobi National Park, Indonesia
}

\author{
Ernik Yuliana ${ }^{1 *}$, Adi Winata ${ }^{2}$, Hasan Eldin Adimu ${ }^{3}$, Yuni Tri Hewindati ${ }^{4}$, Wibowo A. Djatmiko ${ }^{5}$ \\ ${ }^{1}$ Department of Agriculture, Faculty of Science and Technology, Universitas Terbuka, Pamulang, Tangerang Selatan, Indonesia \\ ${ }^{2}$ Department of Technique, Faculty of Science and Technology, Universitas Terbuka, Pamulang, Tangerang Selatan, Indonesia \\ ${ }^{3}$ Department of Marine Science, Faculty of Fisheries and Animal Husbandry, Universitas Sembilanbelas November, Kolaka, Indonesia \\ ${ }^{4}$ Department of Natural Sciences, Faculty of Science and Technology, Universitas Terbuka, Pamulang, Tangerang Selatan, Indonesia \\ ${ }^{5}$ The Indonesian Tropical Institute (LATIN), Bogor, Indonesia
}

\section{ARTICLE INFO}

Article history:

Received March 19, 2021

Received in revised form December 30, 2021

Accepted January 10, 2022

\section{KEYWORDS:}

diversity,

exploitation rate,

Kaledupa,

mudflats,

reef fish,

Wakatobi

\begin{abstract}
Although frequently described as low-fertility or low-productivity habitat, coastal mudflats serve as important feeding grounds for fish. Many fish species from adjacent coral reefs, seagrass beds, or mangroves foraging periodically in mudflats. Because of this foraging behaviour, some local fishermen are known to utilize the mudflats to catch fish. However, the impact of this catching activities to the ecosystem has not been fully discovered. An examination of the fish community structure and levels of environmental stress had carried out in the mudflat ecosystem of the coast of Kaledupa Island in Wakatobi National Park (WNP), Indonesia. Two mudflat study sites were selected from the shore of Balasuna and Tampara villages located between mangroves and coral reefs. Data were sampled from the fish catch of local fishermen using fish fences (sero) installed in each mudflat area. Fish community structure was analyzed using diversity index and index of relative importance (IRI). ABC curves and species exploitation rate were used to assess the local environmental pressure. A total of 74 fish species were recorded from the mudflats of Kaledupa, which was found to be dominated by reef-associated fish species, comprising 63 species and accounting for $85 \%$ of the total catch. Additionally, although both sites had relatively high reef fish diversity, the obtained Clarke's W-statistic values were approximately 0 , indicating that the local fish communities presented moderate levels of disturbance. Three out of five fish species with the highest IRI values were found to be over-exploited, namely Siganus canaliculatus, Lethrinus ornatus, and Lethrinus variegatus.
\end{abstract}

\section{Introduction}

Mudflats are described as low-fertility or lowproductivity (Fanjul et al. 2011) coastal wetlands. At the other hand, coastal mudflats serve as important feeding grounds for fish when the high tide inundates them (Lee et al. 2013). Because of their shallow water column, the benthic process plays a more important role than the pelagic process in this ecosystem Furthermore, water substrates are essential for holding inorganic nutrients originating from the ocean and land runoff (Fanjul et al. 2011). Some fish species are periodically foraging for food in mudflats. The local inhabitants of Kaledupa Island, one of the largest islands in Wakatobi National Park (WNP),

\footnotetext{
* Corresponding Author

E-mail Address: ernik@ecampus.ut.ac.id
}

take advantage of these foraging patterns to catch a large number of fish. Usually, fishermen from the island catch coral reef or pelagic fish using fish fences (locally known as sero) or gill nets. Unfortunately, these methods for catching fish carry the risk of bycatch (i.e., the unintentional capture of non-target species, such as non-commercial or inedible fish), and this can threaten the sustainability of marine resources. Already, some shallow-water habitats in Wakatobi National Park have been adversely affected by the activities of the increasing human population (Azhar et al. 2019).

The WNP is a marine conservation area in Indonesia, officially established on July 30, 1996. WNP extends over an area of $1,390,000$ ha, $97 \%$ of which is sea (Elliott et al. 2001), and, on account of its size, is included in the large-scale conservation category (Ban et al. 2017), and is at the center of the world biodiversity 
(Madduppa et al. 2020). Kaledupa Island is one of the islands in the WNP territory, and like the other islands in its vicinity (Wangi-wangi, Tomia, dan Binongko), it is characterized by mangrove ecosystems and mudflats. These habitats provide a protective environment for coral reefs, seagrass beds, and another ecosystems (Azhar et al. 2019) includes mudflats.

Fish diversity and sustainability in the WNP have received considerable attention since the fundamental objective of this park is to protect marine biodiversity. Fish diversity is an important indicator of fish stock security from which an acceptable level of fishing can be set (Yuliana et al. 2019), and thus studying reef fish diversity is necessary to identify the health status of coral reef ecosystems (Pet-Soede et al. 2001). In this case, abundance and biomass are two important indices for measuring reef fish diversity (Ault et al. 2013).

The present study aimed to analyze the fish community structure in the mudflats of Kaledupa Island. The analyses included the estimation of fish species composition and diversity indices, along with the assessment of the environmental stress suffered by local fish communities using $A B C$ curves and by calculating the rate of exploitation of certain species.

\section{Materials and Methods}

\subsection{Study Site}

This research was conducted in the water bodies adjacent to Kaledupa Island at two different sites: (a) Balasuna Village, located on the east side of the island, and (b) Tampara Village on the west side (Figure 1). The two villages were selected as two representative regions of this island, as they have different conditions; for example, Balasuna faces relatively closed waters originating from a small strait between Kaledupa Island and Hoga Island, while Tampara faces more open waters.

\subsection{Collecting Data}

Data were collected over a period of 6 months and comprised observations of the fishermen's catches collected from the fish fences set in mudflat areas at the front of mangrove forests. The species, number of individuals, their biomass, and catch amount and composition were recorded.

\subsection{Data Analyzed}

The species composition was analyzed using the index of relative importance (IRI) (Hu et al. 2019).
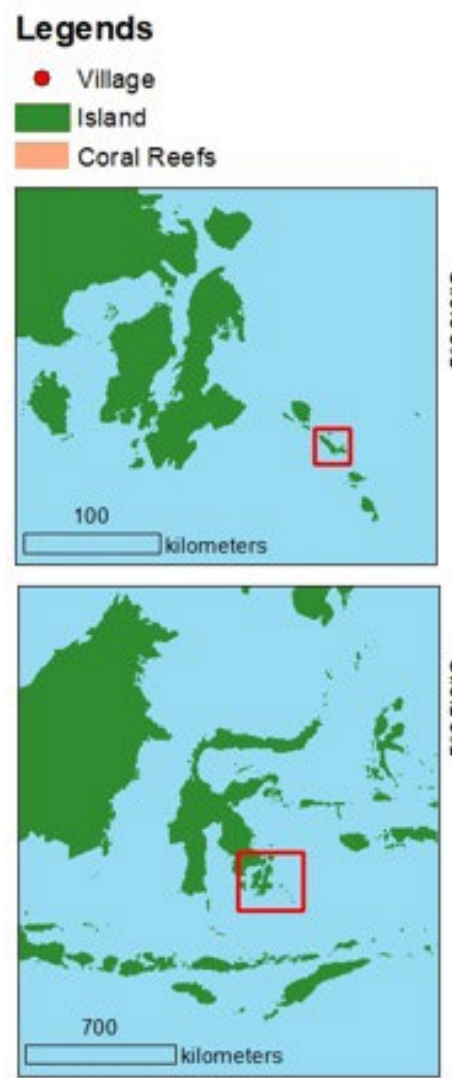

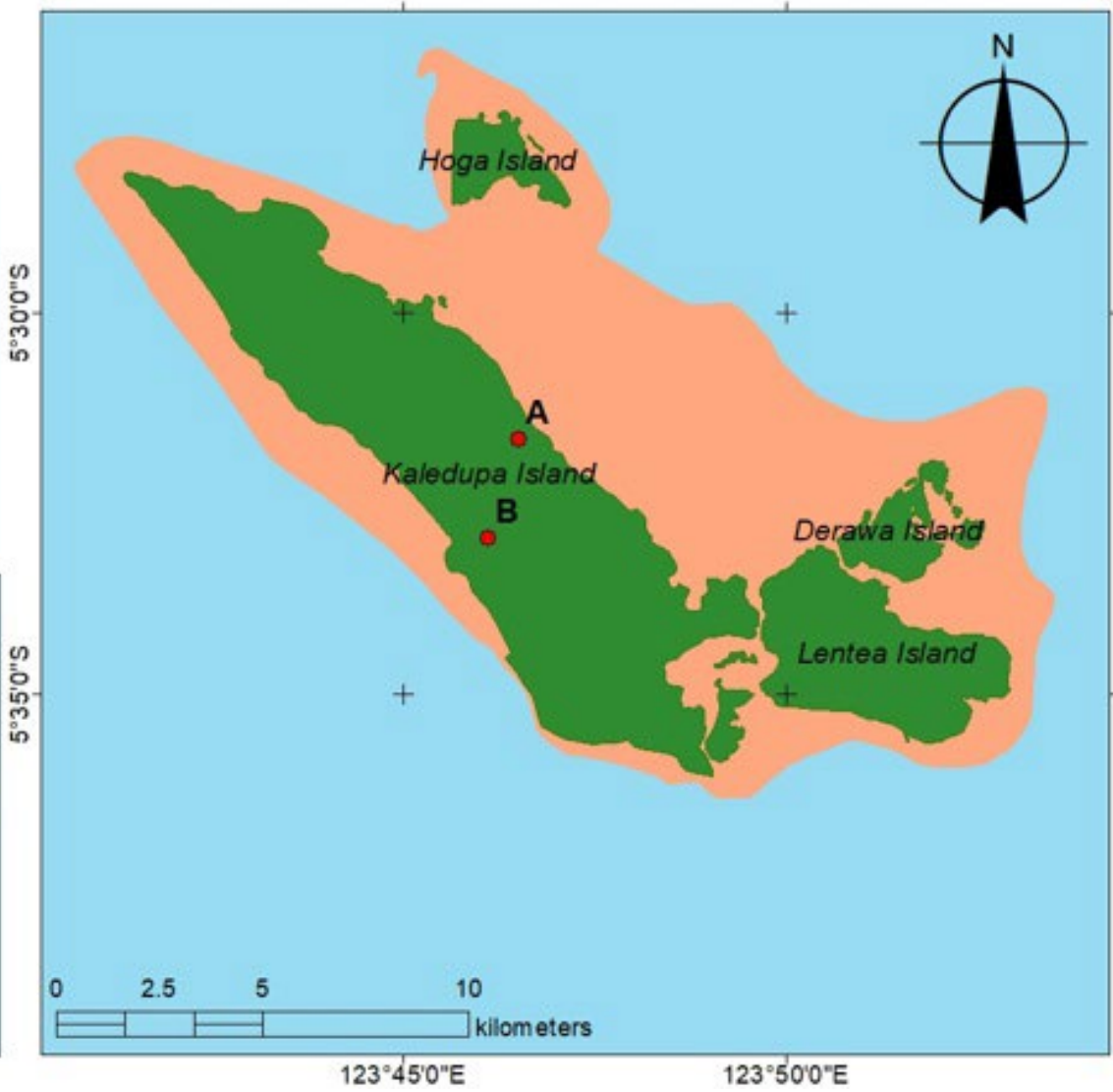

Figure 1. The research sites in Kaledupa Island: Balasuna Village (A) and Tampara Village (B) within Wakatobi National Park's territory, South-east Sulawesi, Indonesia 
Fish diversity analysis was based on the indices of species diversity, evenness, and richness.

The species diversity index ( $\left.\mathrm{H}^{\prime}\right)$ was measured using the following formula by Shannon and Wiener (Odum 1971):

$$
H^{\prime}=p_{i} \ln p_{i}
$$

Where $p_{i}$ is the proportion or the relative abundance ( $\mathrm{ni} / \mathrm{N}$ ) of a given species, ni is the number of individuals of that specified species (i), and $\mathrm{N}$ is the total number of individuals in a given sample.

The species evenness index (E) was calculated according to the following equation (Pielou 1966):

$$
E=\frac{H^{\prime}}{\ln S}
$$

Where $H^{\prime}$ is the species diversity index, and $S$ represents the number of species in a community.

The community similarity index (IS) was determined according to Jaccard's formula, as represented in the following equation:

$$
I S=\frac{a}{\mathrm{a}+\mathrm{b}+\mathrm{c}}
$$

Where a represents the number of individuals in location $\mathrm{A}, \mathrm{b}$ is the number of individuals in location $B$, and $c$ is the number of individuals in both locations (A and $B$ ).

The abundance-biomass comparison (ABC) curve was used to assess the level of environmental disturbance. In this method, species are ranked according to their biomass and abundance, and then plotted along the log-transformed x-axis (Di Bagno et al. 2020). Clarke's W-statistic was calculated using the equation (Meire and Dereu 1990):

$$
W \text {-statistic }=\frac{\sum_{i=1}^{n}\left(\mathrm{~B}_{i}-\mathrm{A}_{i}\right)}{N}
$$

Where $B_{i}$ and $A_{i}$ refer to the biomass and abundance of species $i$, respectively. Values of the $\mathrm{W}$-statistic range from -1 to +1 , indicating the state of the environment from severely disturbed $(W=-1)$ to pristine $(W=+1)$; hence, values close to 0 indicate moderate disturbance (Clarke 1990; Yemane et al. 2005; Di Bagno et al. 2020).

The index of relative importance (IRI) was calculated with the following equation ( $\mathrm{Hu}$ et al. 2019):

$$
I R I=\left(N_{i}+W_{i}\right) \times F_{i}
$$

Where $N_{i}$ represents the relative abundance, $W i$ represents the relative biomass, and $F_{i}$ represents the relative frequency.
The fish exploitation rate (Ex) was calculated based on mortality estimation. Natural mortality estimation was carried out using the FISAT II software with Pauly's M Equation method, total mortality was measured with the long-convertedcatch curve method (Pauly 1983; Gayanilo et al. 2005) fishing mortality rate was determined using the relationship $F=Z-M$. Finally, the rate of exploitation was determined using the following equation:

$$
E x=\frac{F}{Z}
$$

Where $F$ represents fishing mortality, and $Z$ represents total mortality. The rational and sustainable exploitation rate (Ex) for a body of water should be under the value of 0.5 (Sparre and Venema 1998).

\section{Results}

\subsection{Fish Diversity}

A total of 74 fish species were identified from the sampling of local fishermen's catches at the two study sites (Balasuna and Tampara). Furthermore, 63 of those species consisted of reef-associated fish, and the remaining 11 species were pelagic and demersal fish (Table 1). It is evident that reef fish represent a larger proportion than any other group (Figure 2).

Likewise, the five species with the highest IRI were all reef-associated fish. Although three of these species are members of Lethrinidae, the highest IRI belongs to Siganus canaliculatus (Siganidae), with a value of 400.03 (Table 2). Lethrinidae, along with Mullidae and Scaridae, were the most represented families, with each recording six species (Table 1 ).

\subsection{Comparison between Two Sites}

The two study sites recorded almost similar numbers of species, with 58 species in Balasuna and 59 species in Tampara (Table 3); however, only 44 of the 74 species were present in both sites (Table 4). Furthermore, the Shannon-Wiener index of diversity values and species evenness index values at the two sites were also similar (H': 2.89 and 2.93, and E: 0.71 and 0.72 , for Balasuna and Tampara respectively). However, the Tampara site recorded a greater number of individuals, as well as larger biomass values (Table 3 ). These differences are reflected in the IS value of $59.46 \%$, meaning that the similarity of the fish community structures of the two locations is only $60 \%$ (Table 3 ). 
Table 1. The species composition and its distribution in the sampling sites

\begin{tabular}{|c|c|c|c|c|c|c|c|c|}
\hline Family/species & Common name & Fish group & $\begin{array}{l}\text { Balasuna } \\
\text { site }\end{array}$ & $\begin{array}{l}\text { Tampar } \\
\text { site }\end{array}$ & $\begin{array}{l}\text { Number of } \\
\text { Individuals }\end{array}$ & Frequency & $\begin{array}{l}\text { Biomass } \\
\quad(\mathrm{g})\end{array}$ & $\begin{array}{l}\text { Index of } \\
\text { relative } \\
\text { importance } \\
\text { (IRI) }\end{array}$ \\
\hline \multicolumn{9}{|l|}{ Acanthuridae } \\
\hline $\begin{array}{l}\text { Acanthurus } \\
\text { lineatus }\end{array}$ & $\begin{array}{l}\text { Lined } \\
\quad \text { surgeonfish }\end{array}$ & reef-associated & + & - & 2 & 1 & 140 & 140 \\
\hline $\begin{array}{l}\text { Acanthurus } \\
\text { nigricauda }\end{array}$ & $\begin{array}{l}\text { Epaulette } \\
\quad \text { surgeonfish }\end{array}$ & reef-associated & + & - & 4 & 2 & 347 & 347 \\
\hline Naso lituratus & $\begin{array}{l}\text { Orangespine } \\
\text { unicornfish }\end{array}$ & reef-associated & - & + & 4 & 1 & 259 & 259 \\
\hline \multicolumn{9}{|l|}{ Atherinidae } \\
\hline $\begin{array}{r}\text { Atherinomorus } \\
\text { duodecimalis }\end{array}$ & $\begin{array}{l}\text { Tropical } \\
\text { silverside }\end{array}$ & demersal & - & + & 20 & 3 & 288 & 288 \\
\hline $\begin{array}{l}\text { Atherinomorus } \\
\text { lacunasus }\end{array}$ & $\begin{array}{l}\text { Wide-banded } \\
\text { hardyhead } \\
\text { silverside }\end{array}$ & reef-associated & - & + & 12 & 2 & 181 & 181 \\
\hline Balistidae & & & & & & & & 252 \\
\hline $\begin{array}{l}\text { Balistapus } \\
\text { undulatus }\end{array}$ & $\begin{array}{l}\text { Orange-lined } \\
\text { triggerfish }\end{array}$ & reef-associated & + & - & 2 & 2 & 252 & \multirow[t]{2}{*}{225} \\
\hline $\begin{array}{c}\text { Rhinecanthus } \\
\text { verrucosus }\end{array}$ & $\begin{array}{l}\text { Blackbelly } \\
\text { triggerfish }\end{array}$ & reef-associated & + & - & 1 & 1 & 225 & \\
\hline \multicolumn{9}{|l|}{ Belonidae } \\
\hline $\begin{array}{l}\text { Tylosurus } \\
\text { crocodilus }\end{array}$ & $\begin{array}{l}\text { Hound } \\
\text { needlefish }\end{array}$ & reef-associated & + & + & 17 & 6 & 2,220 & 2,220 \\
\hline \multicolumn{9}{|l|}{ Carangidae } \\
\hline $\begin{array}{l}\text { Carangoides } \\
\text { chrysophrys }\end{array}$ & $\begin{array}{l}\text { Longnose } \\
\text { trevally }\end{array}$ & reef-associated & + & - & 3 & 1 & 636 & 636 \\
\hline $\begin{array}{l}\text { Carangoides } \\
\text { ferdau }\end{array}$ & Blue trevally & reef-associated & + & + & 21 & 5 & 2,225 & 2,225 \\
\hline Caranx hippos & Crevalle jack & reef-associated & - & + & 2 & 1 & 219 & 0.02 \\
\hline Caranx tille & Tille trevally & reef-associated & + & + & 28 & 7 & 2,175 & 2.43 \\
\hline \multicolumn{9}{|l|}{ Chaetodontidae } \\
\hline $\begin{array}{l}\text { Chaetodon } \\
\text { vagabundus }\end{array}$ & $\begin{array}{l}\text { Vagabond } \\
\text { butterflyfish }\end{array}$ & reef-associated & + & + & 9 & 7 & 695 & 0.71 \\
\hline \multicolumn{9}{|l|}{ Diodontidae } \\
\hline $\begin{array}{l}\text { Diodon } \\
\quad \text { holocanthus }\end{array}$ & $\begin{array}{l}\text { Longspined } \\
\text { porcupinefish }\end{array}$ & reef-associated & + & + & 15 & 8 & 5,062 & 3.75 \\
\hline \multicolumn{9}{|l|}{ Eleotridae } \\
\hline $\begin{array}{r}\text { Oxyeleotris } \\
\text { lineolata }\end{array}$ & Sleepy cod & demersal & - & + & 20 & 1 & 2,424 & 0.20 \\
\hline \multicolumn{9}{|l|}{ Ephippidae } \\
\hline $\begin{array}{l}\text { Platax pinatus } \\
\text { Platax }\end{array}$ & $\begin{array}{l}\text { Dusky batfish } \\
\text { Orbicular }\end{array}$ & reef-associated & + & + & 13 & 5 & 1,348 & 0.94 \\
\hline $\begin{array}{l}\text { orbicularis } \\
\text { Exocoetidae }\end{array}$ & batfish & reef-associated & + & + & 10 & 4 & 405 & 0.31 \\
\hline $\begin{array}{c}\text { Exocoetidae } \\
\text { Cypselurus } \\
\text { naresii }\end{array}$ & $\begin{array}{l}\text { Pharao } \\
\text { flyingfish }\end{array}$ & pelagic & + & + & 18 & 8 & 1,368 & 1.69 \\
\hline \multicolumn{9}{|l|}{ Gerreidae } \\
\hline $\begin{array}{l}\text { Gerres } \\
\quad \text { filamentosus }\end{array}$ & $\begin{array}{l}\text { Whipfin silver- } \\
\text { biddy }\end{array}$ & demersal & + & + & 60 & 14 & 5,927 & 11.11 \\
\hline Gerres oyena & $\begin{array}{l}\text { Common silver- } \\
\text { biddy }\end{array}$ & reef-associated & + & - & 2 & 1 & 363 & 0.05 \\
\hline \multicolumn{9}{|l|}{ Haemulidae } \\
\hline $\begin{array}{l}\text { Diagramma } \\
\text { melanacrum }\end{array}$ & Blackfin slatey & bentho-pelagic & + & - & 4 & 2 & 566 & 0.17 \\
\hline $\begin{array}{l}\text { Plectorhinchus } \\
\quad \text { lineatus }\end{array}$ & $\begin{array}{l}\text { Yellowbanded } \\
\text { sweetlips }\end{array}$ & reef-associated & + & + & 67 & 12 & 7,183 & 10.46 \\
\hline $\begin{array}{l}\text { Pomadasys } \\
\text { furcatus }\end{array}$ & Banded grunter & reef-associated & + & + & 13 & 4 & 964 & 0.70 \\
\hline
\end{tabular}


Table 1. Continued

\begin{tabular}{|c|c|c|c|c|c|c|c|c|}
\hline Family/species & Common name & Fish group & $\begin{array}{l}\text { Balasuna } \\
\text { site }\end{array}$ & $\begin{array}{l}\text { Tampar } \\
\text { site }\end{array}$ & $\begin{array}{l}\text { Number of } \\
\text { Individuals }\end{array}$ & Frequency & $\begin{array}{l}\text { Biomass } \\
(\mathrm{g})\end{array}$ & $\begin{array}{l}\text { Index of } \\
\text { relative } \\
\text { importance } \\
\text { (IRI) }\end{array}$ \\
\hline \multicolumn{9}{|l|}{ Hemiramphidae } \\
\hline Hemiramphus far & $\begin{array}{l}\text { Black-barred } \\
\text { halfbeak }\end{array}$ & reef-associated & + & - & 8 & 3 & 677 & 0.39 \\
\hline $\begin{array}{l}\text { Hyporhamphus } \\
\text { quoyi }\end{array}$ & Quoy's garfish & pelagic-neritic & + & + & 21 & 6 & 819 & 0.91 \\
\hline $\begin{array}{l}\text { Hyporhamphus } \\
\text { affinis }\end{array}$ & $\begin{array}{l}\text { Tropical } \\
\text { halfbeak }\end{array}$ & reef-associated & + & - & 2 & 2 & 227 & 0.08 \\
\hline \multicolumn{9}{|l|}{ Holocentridae } \\
\hline $\begin{array}{l}\text { Myripristis } \\
\text { berndti }\end{array}$ & $\begin{array}{l}\text { Blotcheye } \\
\text { soldierfish }\end{array}$ & reef-associated & - & + & 1 & 1 & 52 & 0.01 \\
\hline $\begin{array}{l}\text { Myriptristis } \\
\text { amaena }\end{array}$ & $\begin{array}{l}\text { Brick } \\
\text { soldierfish }\end{array}$ & reef-associated & - & + & 2 & 1 & 72 & 0.01 \\
\hline $\begin{array}{l}\text { Sargocentron } \\
\text { cornutum }\end{array}$ & $\begin{array}{l}\text { Threespot } \\
\text { squirrelfish }\end{array}$ & reef-associated & - & + & 1 & 1 & 43 & 0.01 \\
\hline \multicolumn{9}{|l|}{ Labridae } \\
\hline Cheilio inermis & Cigar wrasse & reef-associated & - & + & 2 & 1 & 57 & 0.01 \\
\hline $\begin{array}{l}\text { Choerodon } \\
\text { anchorago }\end{array}$ & $\begin{array}{l}\text { Orange-dotted } \\
\text { tuskfish }\end{array}$ & reef-associated & + & + & 114 & 18 & 8,989 & 23.56 \\
\hline $\begin{array}{l}\text { Choerodon } \\
\text { cephalotes }\end{array}$ & Purple tuskfish & reef-associated & + & - & 1 & 1 & 237 & 0.03 \\
\hline $\begin{array}{l}\text { Thalassoma } \\
\text { hardwicke }\end{array}$ & Sixbar wrasse & reef-associated & + & - & 1 & 1 & 47 & 0.01 \\
\hline \multicolumn{9}{|l|}{ Leiognathidae } \\
\hline Gazza dentex & $\begin{array}{l}\text { Ovoid } \\
\text { toothpony }\end{array}$ & demersal & + & + & 12 & 4 & 1,075 & 0.61 \\
\hline \multicolumn{9}{|l|}{ Lethrinidae } \\
\hline $\begin{array}{l}\text { Lethrinus } \\
\text { erythropterus }\end{array}$ & $\begin{array}{l}\text { Longfin } \\
\text { emperor }\end{array}$ & reef-associated & + & + & 11 & 5 & 2,480 & 1.26 \\
\hline $\begin{array}{l}\text { Lethrinus } \\
\text { genivittatus }\end{array}$ & $\begin{array}{l}\text { Longspin } \\
\text { emperor }\end{array}$ & reef-associated & + & + & 6 & 4 & 646 & 0.33 \\
\hline Lethrinus harak & $\begin{array}{l}\text { Thumbprint } \\
\text { emperor }\end{array}$ & reef-associated & + & + & 505 & 36 & 73,179 & 295.42 \\
\hline Lethrinus lentjan & $\begin{array}{l}\text { Pink ear } \\
\text { emperor }\end{array}$ & reef-associated & - & + & 4 & 1 & 550 & 0.04 \\
\hline Lethrinus ornatus & Ornate emperor & reef-associated & + & + & 172 & 19 & 26,978 & 52.73 \\
\hline $\begin{array}{l}\text { Lethrinus } \\
\text { variegatus } \\
\text { Lutianidae }\end{array}$ & $\begin{array}{l}\text { Slender } \\
\text { emperor }\end{array}$ & reef-associated & + & + & 210 & 18 & 11,864 & 38.07 \\
\hline $\begin{array}{l}\text { Lutjanus } \\
\text { fulviflamma }\end{array}$ & Dory snapper & reef-associated & + & + & 15 & 7 & 2,539 & 1.78 \\
\hline $\begin{array}{l}\text { Lutjanus } \\
\text { monostigma }\end{array}$ & $\begin{array}{l}\text { One-spot } \\
\text { snapper }\end{array}$ & reef-associated & - & + & 7 & 3 & 928 & 0.22 \\
\hline $\begin{array}{l}\text { Lutjanus } \\
\text { quinquelineatus }\end{array}$ & $\begin{array}{r}\text { Five-lined } \\
\text { snapper }\end{array}$ & reef-associated & + & - & 3 & 1 & 294 & 0.05 \\
\hline $\begin{array}{l}\text { Mugilidae } \\
\text { Ellochelon } \\
\text { vaigiensis }\end{array}$ & $\begin{array}{l}\text { Squaretail } \\
\text { mullet }\end{array}$ & reef-associated & + & + & 21 & 6 & 4,707 & 2.40 \\
\hline \multicolumn{9}{|l|}{ Mullidae } \\
\hline $\begin{array}{l}\text { Mulloidichthys } \\
\text { martinicus }\end{array}$ & Yellow goatfish & reef-associated & + & + & 4 & 2 & 492 & 0.12 \\
\hline $\begin{array}{l}\text { Mulloidichthys } \\
\text { flavolineatus }\end{array}$ & $\begin{array}{l}\text { Yellowstripe } \\
\text { goatfish }\end{array}$ & reef-associated & + & + & 130 & 25 & 10,624 & 40.94 \\
\hline $\begin{array}{l}\text { Parupeneus } \\
\text { barberinus }\end{array}$ & $\begin{array}{l}\text { Dash-and-dot } \\
\text { goatfish }\end{array}$ & reef-associated & + & + & 36 & 5 & 2,615 & 2.21 \\
\hline $\begin{array}{l}\text { Parupeneus } \\
\text { crassilabris }\end{array}$ & $\begin{array}{l}\text { Ray-finned } \\
\text { fishes }\end{array}$ & reef-associated & + & + & 71 & 15 & 3,838 & 10.73 \\
\hline $\begin{array}{l}\text { Parupeneus } \\
\text { cyclostomus }\end{array}$ & $\begin{array}{l}\text { Gold-saddle } \\
\text { goatfish }\end{array}$ & reef-associated & + & + & 90 & 14 & 6,976 & 14.91 \\
\hline Upeneus tragula & $\begin{array}{l}\text { Freckled } \\
\text { goatfish }\end{array}$ & reef-associated & + & + & 8 & 4 & 901 & 0.53 \\
\hline
\end{tabular}


Table 1. Continued

\begin{tabular}{|c|c|c|c|c|c|c|c|c|}
\hline Family/species & Common name & Fish group & $\begin{array}{l}\text { Balasuna } \\
\text { site }\end{array}$ & $\begin{array}{l}\text { Tampar } \\
\text { site }\end{array}$ & $\begin{array}{l}\text { Number of } \\
\text { Individuals }\end{array}$ & Frequency & $\begin{array}{l}\text { Biomass } \\
\quad(\mathrm{g})\end{array}$ & $\begin{array}{l}\text { Index of } \\
\text { relative } \\
\text { importance } \\
\text { (IRI) }\end{array}$ \\
\hline \multicolumn{9}{|l|}{ Nemipteridae } \\
\hline Scolopsis aurata & $\begin{array}{l}\text { Yellowstripe } \\
\text { monocle } \\
\text { bream }\end{array}$ & reef-associated & + & - & 1 & 1 & 255 & 0.03 \\
\hline Scolopsis ciliata & $\begin{array}{l}\text { Saw-jawed } \\
\text { monocle } \\
\text { bream }\end{array}$ & reef-associated & + & + & 116 & 18 & 7,050 & 19.93 \\
\hline Scolopsis lineata & $\begin{array}{l}\text { Striped } \\
\text { monocle } \\
\text { bream }\end{array}$ & reef-associated & + & + & 55 & 16 & 2,622 & 6.88 \\
\hline $\begin{array}{l}\text { Scolopsis } \\
\quad \text { xenochroa }\end{array}$ & $\begin{array}{l}\text { Oblique-barred } \\
\text { monocle } \\
\text { bream }\end{array}$ & reef-associated & + & + & 12 & 5 & 718 & 0.51 \\
\hline \multicolumn{9}{|l|}{ Paralichthyidae } \\
\hline $\begin{array}{r}\text { Pseudorhombus } \\
\text { dupliocellatus }\end{array}$ & $\begin{array}{l}\text { Ocellated } \\
\text { flounder }\end{array}$ & demersal & - & + & 1 & 1 & 28 & 0.01 \\
\hline \multicolumn{9}{|l|}{ Platycephalidae } \\
\hline $\begin{array}{l}\text { Cymbacephalus } \\
\text { beauforti }\end{array}$ & Crocodile fish & reef-associated & + & + & 5 & 3 & 2,446 & 0.52 \\
\hline Inegocia japonica & $\begin{array}{l}\text { Japanese } \\
\text { flathead }\end{array}$ & demersal & - & + & 0 & 1 & 0 & 0.03 \\
\hline \multicolumn{9}{|l|}{ Plotosidae } \\
\hline $\begin{array}{l}\text { Plotosus canius } \\
\text { Scaridae }\end{array}$ & Gray eel-catfish & \multicolumn{4}{|c|}{ Scaridae } & 1 & 214 & 0.02 \\
\hline $\begin{array}{l}\text { Leptocerus } \\
\text { vaigiensis }\end{array}$ & $\begin{array}{l}\text { Marbled } \\
\text { parrotfish }\end{array}$ & reef-associated & + & + & 46 & 11 & 5,629 & 7.70 \\
\hline Scarus croicensis & $\begin{array}{l}\text { Striped } \\
\text { parrotfish }\end{array}$ & reef-associated & - & + & 2 & 1 & 326 & 0.02 \\
\hline Scarus dimidiatus & $\begin{array}{l}\text { Yellowbarred } \\
\text { parrotfish }\end{array}$ & reef-associated & + & + & 21 & 8 & 3,014 & 2.49 \\
\hline Scarus ghobban & $\begin{array}{l}\text { Blue-barred } \\
\text { parrotfish }\end{array}$ & reef-associated & + & - & 2 & 1 & 450 & 0.06 \\
\hline Scarus psittacus & $\begin{array}{l}\text { Common } \\
\text { parrotfish }\end{array}$ & reef-associated & + & + & 4 & 2 & 544 & 0.13 \\
\hline Scarus quoyi & $\begin{array}{l}\text { Quoy's } \\
\text { parrotfish }\end{array}$ & reef-associated & + & + & 5 & 2 & 592 & 0.15 \\
\hline Scarus schlegeli & $\begin{array}{l}\text { Yellowband } \\
\text { parrotfish }\end{array}$ & reef-associated & + & + & 23 & 7 & 1,286 & 1.44 \\
\hline \multicolumn{9}{|l|}{ Serranidae } \\
\hline $\begin{array}{l}\text { Epinephelus } \\
\text { bleekeri } \\
\text { Siganidae }\end{array}$ & $\begin{array}{l}\text { Duskytail } \\
\text { grouper }\end{array}$ & demersal & + & + & 6 & 4 & 1,403 & 0.53 \\
\hline $\begin{array}{l}\text { Siganidae } \\
\text { Siganus } \\
\text { canaliculatus }\end{array}$ & $\begin{array}{l}\text { White-spotted } \\
\text { spinefoot }\end{array}$ & reef-associated & + & + & 808 & 40 & 71,892 & 400.02 \\
\hline Siganus guttatus & $\begin{array}{l}\text { Orange-spotted } \\
\text { spinefoot }\end{array}$ & reef-associated & + & + & 64 & 11 & 21,014 & 18.09 \\
\hline Siganus spinus & Little spinefoot & reef-associated & + & + & 161 & 16 & 8,472 & 26.29 \\
\hline Siganus virgatus & $\begin{array}{l}\text { Barhead } \\
\text { spinefoot }\end{array}$ & reef-associated & + & + & 45 & 7 & 2,212 & 3.29 \\
\hline \multicolumn{9}{|l|}{ Sphyraenidae } \\
\hline $\begin{array}{l}\text { Sphyraena } \\
\text { barracuda }\end{array}$ & Great barracuda & reef-associated & + & + & 7 & 4 & 1,707 & 0.64 \\
\hline $\begin{array}{r}\text { Sphyraena } \\
\text { obtusata }\end{array}$ & $\begin{array}{l}\text { Obtuse } \\
\text { barracuda }\end{array}$ & reef-associated & + & - & 1 & 1 & 76 & 0.02 \\
\hline \multicolumn{9}{|l|}{ Toxotidae } \\
\hline Toxotes jaculatrix & $\begin{array}{l}\text { Banded } \\
\text { archerfish }\end{array}$ & reef-associated & + & + & 36 & 6 & 3,460 & 2.51 \\
\hline $\begin{array}{l}\text { Zanclidae } \\
\text { Zanclus cornutus }\end{array}$ & Moorish idol & reef-associated & - & + & 1 & 1 & 34 & 0.01 \\
\hline
\end{tabular}

+: present, -: absent 


\subsection{Environmental Disturbance}

The water quality of the research location was classified as good (Table 5 ). Of the three water quality parameters measured, two ( $\mathrm{pH}$ and temperature)

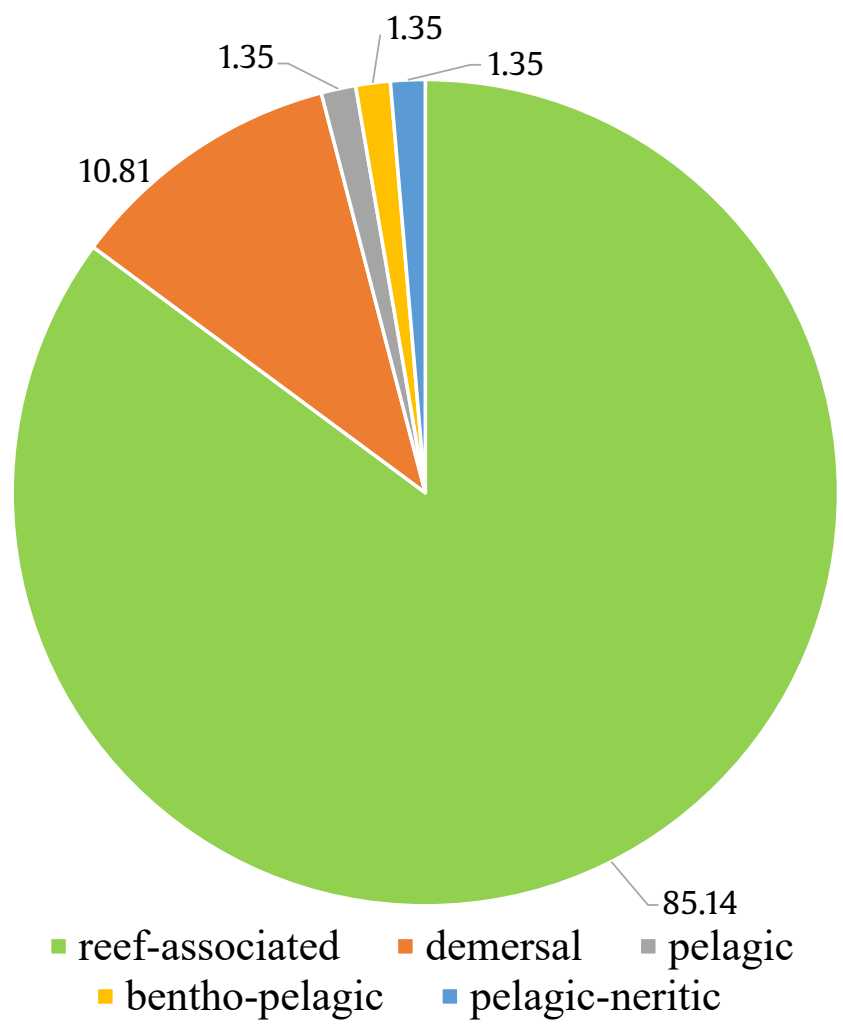

Figure 2. Reef fish proportion in the total fish catches (\%)

Table 2. Five fish species with the highest IRI

\begin{tabular}{llr}
\hline Fish species & Common Name (English) & \multicolumn{1}{c}{ IRI } \\
\hline Siganus canaliculatus & White-spotted spinefoot & 400.03 \\
Lethrinus harak & Thumbprint emperor & 295.44 \\
Lethrinus ornatus & Ornate emperor & 52.73 \\
Mulloidichthys & Yellowstripe goatfish & 40.95 \\
$\quad$ flavolineatus & & \\
Lethrinus variegatus & Slender emperor & 38.07 \\
\hline
\end{tabular}

were in accordance with the Water Quality Standards for Marine Biota (Indonesian State Minister of the Environment Decree no. 51/2004).

However, the $A B C$ curves obtained for the two study sites appeared to be almost entirely overlapped between the abundance line and biomass line, implying that the fish communities of both locations were moderately disturbed (Figure 3 and 4). Furthermore, this conclusion is confirmed by the $\mathrm{W}$-statistic value, which was approximately $0(\mathrm{~W}=$ 0.0136 for Balasuna, and $\mathrm{W}=-\mathbf{0 . 0 0 0 2}$ for Tampara).

One form of environmental pressure in aquatic environments is the over-exploitation of target fish communities. Such effects can be detected by measuring the fish exploitation rate, obtained from the analysis of mortality based on the size of the fish catch. The analysis of the exploitation rate revealed that three of the five fish species with the highest IRI values were over-exploited (Table 6), namely $S$. canaliculatus $(\mathrm{Ex}=0.55)$, Lethrinus ornatus $(\mathrm{Ex}=0.65)$, and $L$. variegatus $(\mathrm{Ex}=0.57)$. These results indicate that Kaledupa waters at both study sites have been over-exploited.

\section{Discussion}

Mudflats, which are part of coastal ecosystems, are occupied by fish species that are not specifically associated with these areas. Our study revealed that 63 spp. (85.13\%) out of 74 spp. recorded were categorized as reef-associated fish. Other study on

Table 4. Matrix of the fishes' presence in Balasuna and Tampara

\begin{tabular}{llrr}
\hline \multirow{2}{*}{ Fish species presence in the study sites } & \multicolumn{2}{c}{ Tampara } \\
\cline { 2 - 3 } Bresence Absence & & P4(a) & $14(\mathrm{~b})$ \\
\hline \multirow{2}{*}{ Balasuna } & Presence & $15(\mathrm{c})$ & $0(\mathrm{~d})$ \\
\hline
\end{tabular}

Table 3. The numbers of fish species, numbers of fish individuals, biomass, and species diversity in Balasuna and Tampara

\begin{tabular}{lllcccc}
\hline Study sites & $\begin{array}{l}\text { Total number } \\
\text { of species }\end{array}$ & $\begin{array}{l}\text { Total Number } \\
\text { of Individuals }\end{array}$ & $\begin{array}{c}\text { Total } \\
\text { Biomass }(\mathrm{g})\end{array}$ & $\begin{array}{c}\text { Species } \\
\text { diversity }\left(H^{\prime}\right)\end{array}$ & $\begin{array}{c}\text { Species } \\
\text { evenness }(E)\end{array}$ & $\begin{array}{c}\text { Community } \\
\text { similarity }(I S)\end{array}$ \\
\hline Balasuna & 58 & 1,393 & 162,005 & 2.89 & 0.71 \\
\hline Tampara & 59 & 1,837 & 171,397 & 2.93 & 0.72 & $59.46 \%$ \\
\hline
\end{tabular}

Table 5. Assessments of water quality in Tampara and Balasuna

\begin{tabular}{|c|c|c|c|c|c|c|c|c|}
\hline \multirow{2}{*}{ Water quality } & \multicolumn{4}{|c|}{ Tampara } & \multicolumn{4}{|c|}{ Balasuna } \\
\hline & $\mathrm{U}$ & $\mathrm{M}$ & $\mathrm{L}$ & Mean values & $\mathrm{U}$ & $\mathrm{M}$ & $\mathrm{L}$ & Mean values \\
\hline$\overline{\mathrm{H}}$ & 7.33 & 7.23 & 7.70 & 7.42 & 7.30 & 7.97 & 7.00 & 7.42 \\
\hline Temperature $\left({ }^{\circ} \mathrm{C}\right)$ & 28.67 & 27.67 & 29.33 & 28.56 & 29.00 & 28.3 & 28.70 & 28.67 \\
\hline TDS (mg/L) & 84 & 82 & 86 & 84 & 87 & 86 & 86 & 86 \\
\hline
\end{tabular}

$\mathrm{U}=$ upper (landward) zone, $\mathrm{M}$ = middle zone, $\mathrm{L}$ = lower (seaward) zone 


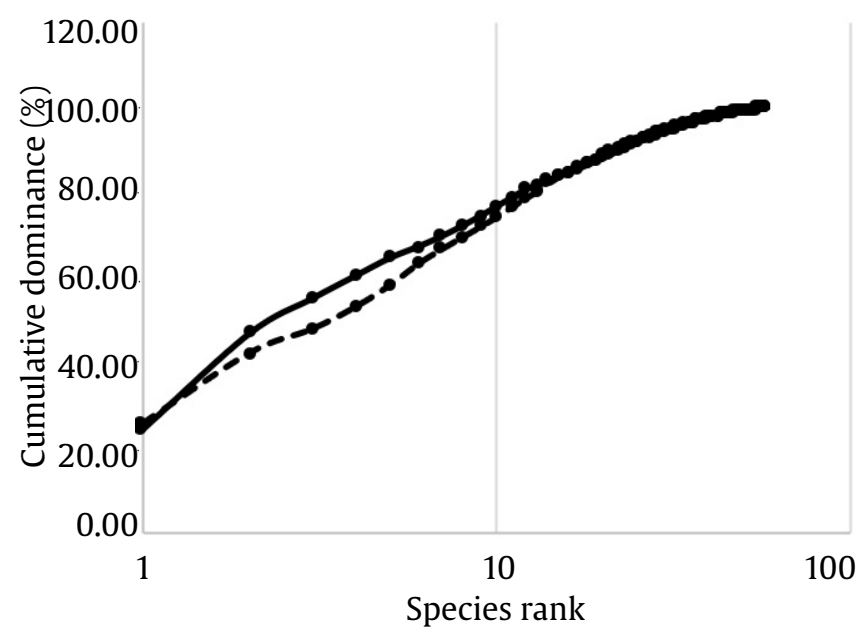

Figure 3. The comparison of abundance-biomass curves in the Balasuna site $(\mathrm{W}=0.0136)$

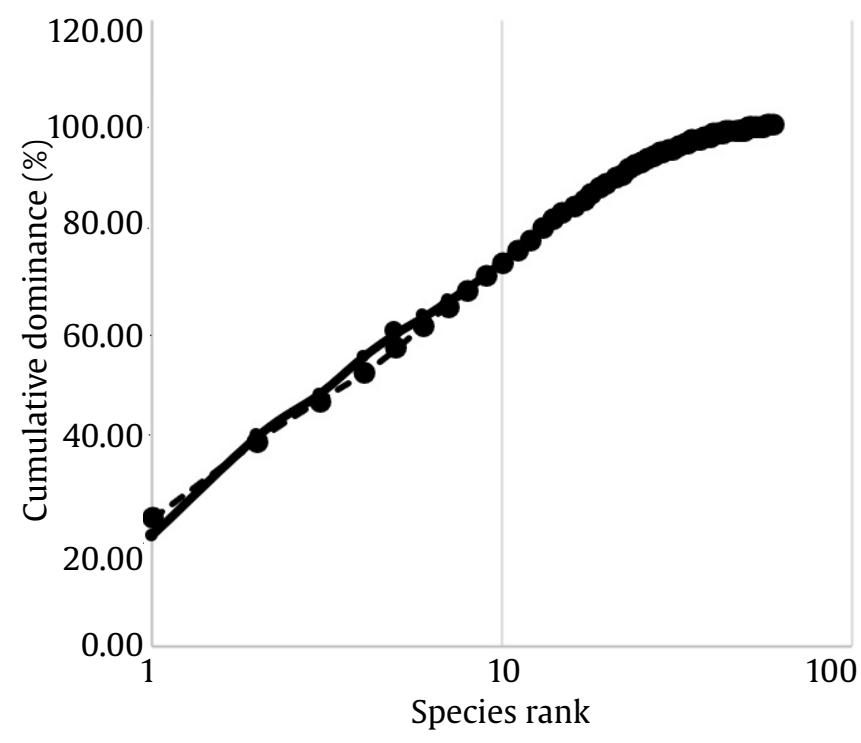

--a--Abundance Biomass

Figure 4. The comparison of abundance-biomass curves in the Tampara site $(\mathrm{W}=-0.0002)$

Table 6. Exploitation rate for five fish species with highest IRI value

\begin{tabular}{|c|c|c|c|c|c|c|c|}
\hline \multirow{2}{*}{ Species } & \multicolumn{3}{|c|}{ Growth parameters } & \multicolumn{3}{|c|}{ Mortality } & \multirow[b]{2}{*}{ Ex } \\
\hline & $\mathrm{L}_{\infty}(\mathrm{cm})$ & $\mathrm{k}\left(\right.$ year $\left.^{-1}\right)$ & $\overline{t_{0} \text { (year) }}$ & $\mathrm{M}\left(\right.$ year $\left.^{-1}\right)$ & $\left(\right.$ year $\left.^{-1}\right)$ & $Z\left(\right.$ year $\left.^{-1}\right)$ & \\
\hline Siganus canaliculatus & 30.76 & 0.74 & -0.22 & 1.20 & 1.48 & 2.68 & 0.55 \\
\hline Lethrinus harak & 31.52 & 0.50 & -0.32 & 0.92 & 0.87 & 1.79 & 0.48 \\
\hline Lethrinus ornatus & 31.52 & 0.55 & $-0,29$ & 0.98 & 1.82 & 2.80 & 0.65 \\
\hline Mulloidichthys flavolineatus & 27.34 & 0.53 & -0.32 & 1.00 & 0.71 & 1.71 & 0.41 \\
\hline Lethrinus variegatus & 21.05 & 0.47 & -0.38 & 0.99 & 1.31 & 2.30 & 0.57 \\
\hline
\end{tabular}

marginal habitats, e.g. near shore habitats around coral reef margins, of Hoga Island of Wakatobi, records many fish species shuttle between areas as the tides change. Some reasons of such shuttling behavior, as suggested, were to avoid larger predator, to access a wider range of foraging areas, and to reduced locomotion costs by exploiting natural tidal flows (Bennett 2010).

The mudflats in our two study sites were located between the mangrove forests and coral reefs. Such situation indicates that there is a close ecological relationship between coral reefs, mangroves, and mudflat ecosystems in Kaledupa Island.

This study revealed that the fish assemblage in the Kaledupa mudflats is dominated by reefassociated fish species. This is related to the nature of the WNP waters, which contain a large coral reef area, even though it has decreased from 2,217 ha to 2,039 ha between 2002 and 2016 (Azhar et al. 2019). This loss of reef coverage is suspected to be caused by destructive fishing activities, such as bombs and poisons (Azhar et al. 2019). Although preventive and monitoring measures are being taken, this decline of coral reef areas is thought to affect fish diversity (PetSoede et al. 2001).

However, relatively high fish diversity was still recorded at the two study sites on Kaledupa Island. The Shannon-Wiener diversity index $\left(H^{\prime}\right)$ values were close to 3 (Table 3 ), which is considered to be moderate to high. As a comparison, $H^{\prime}$ values for mudflat's fish assemblage in Klang, Malaysia ranged from 2.77 to 3.12 (Lee et al. 2013). Meanwhile, the assessment of fish communities in the lower Gianjiang River revealed that the values of the diversity index varied between 1.54 and 2.93 (Hu et al. 2019).

These conditions should be maintained as they greatly contribute to the high fish diversity currently observed in the surrounding area. The findings of this study indicate that fishing activities in Kaledupa are generally under control. Most fishermen in the two villages use traditional and passive fishing gear 
(especially sero) to minimize habitat disturbance and to conserve reef fish stocks. Unfortunately, a small proportion of local fishermen still utilize bombs and poison. The main disturbance to the coral reefs came from human (high and destructive fishing, pollution) and nature (sedimentation and climate change) (Hughes et al. 2012). The exact potential impacts of these threats are still unclear in many cases, thus making effective conservation more difficult (Madduppa et al. 2020).

As previously mentioned, some levels of disturbance occurred at both sites, as confirmed by the obtained $A B C$ curves and Clarke's W-statistic values. Theoretically, $A B C$ curves represent the two states between the " $r$ " and " $k$ " strategies of the evolutionary strategy theory. Positive W-statistic values (approximately +1 ) indicate that the biomass curve lies above the abundance curve, describing an undisturbed state where the k-selected species (with a large body size, but low in numbers) dominate the community, and vice versa (Clarke 1990; Yemane et al. 2005; Di Bagno et al. 2020). The W-statistic values obtained for the two sites were close to 0 , demonstrating that the studied mudflat ecosystems were under moderate disturbance, although Balasuna was slightly less affected than Tampara.

Lies about the centre of the 'Coral Triangle' region, the WNP has a rich and abundant coral reef ecosystem (McMellor and Smith 2013). In turn, Government Regulation of the Republic of Indonesia no. 50/2011 concerning the National Tourism Development Master Plan 2010-2025 has declared the WNP as one of the National Tourism Strategic Areas (Wijaya and Damanik 2020). At the other hand, the rise of marine tourism can leads to coastal marine pollution, either from ship pollution, white (plastic) pollution, domestic waste generated by human activities, including tourism (Zheng dan Liu 2021). Therefore, it is necessary to balance the activities of tourism and fisheries to prevent them from harming each other (Yuliana et al. 2019).

However, pressure on fish communities also originates from fishing activities, as shown by the exploitation rates obtained from several dominant fish species (higher than 0.5), indicating a slightly over-exploited population. As suggested by many authors (e.g. Pauly 1983) optimal exploitation rate $\left(E_{\text {opt }}\right)$ for fishing fish stock is less than or equal to 0.5 .

Although sampling in this study was carried out on the catch of fishermen who used passive fishing gear, there are further fishing pressures arising from other types of fishing gear used around Kaledupa Island. Such situation needs to be resolved, both by Park management and other stakeholders including fishermen. Yuliana et al. (2019) suggest at least three policy recommendations to control over-exploitation, e.g. 1) educate fishers to build their awareness to catch adult fish only, 2) regulate fishing effort by setting mesh size limits of fishnet, 3 ) increase the monitoring of fishing activities.

\section{Acknowledgements}

The authors are grateful to the Universitas Terbuka for providing research funding through the fundamental research scheme. We also thank the authorities of Wakatobi National Park for granting permission to conduct our research at the study site. Finally, we would like to thank the anonymous reviewer for the valuable comments and criticism that contributed to the substantial improvement of the manuscript.

\section{References}

Ault, J.S., Smith, S.G., Bohnsack, J.A., Luo, J., Zurcher, N., McClellan, D.B., Ziegler, T.A., Hallac, D.E., Patterson, M., Feeley, M.W., 2013. Assessing coral reef fish population and community changes in response to marine reserves in the dry tortugas, Florida, USA. Fish. Res. 144, 28-37. https://doi.org/10.1016/j. fishres.2012.10.007

Azhar, A., Damar, A., Bengen, D.G., Atmadipoera, A.S., 2019. Shallow water marine habitat mapping of Kaledupa Island using integrating tradisional ecological knowledge and multispectral image classification. IOP Conference Series: Earth and Environmental Science. 241, 1-10. https://doi.org/10.1088/17551315/241/1/012038

Ban, N.C., Davies, T.E, Aguilera, S.E., Brooks, C., Cox, M., Epstein, G., Evans, L.S., Maxwell, S.M., Nenadovic, M., 2017. Social and ecological effectiveness of large marine protected areas. Glob. Environ. Chang. 43, 8291. https://doi.org/10.1016/j.gloenvcha.2017.01.003

Bennett, W., 2010. Extreme physiology of intertidal fishes of the Wakatobi. in: Clifton, J., Unsworth, R.K.F., Smith, D.J., (Eds.), Marine Research and Conservation in the Coral Triangle: the Wakatobi National Park. Operation Wallacea and Gov't of Wakatobi Regency, Wakatobi. pp. 108-124.

Clarke, K.R., 1990. Comparisons of dominance curves. J. Exp. Mar. Bio. Ecol. 138, 143-157. https://doi. org/10.1016/0022-0981(90)90181-B

Di Bagno, E., Battisti, C., Zullo, F., Amori, G., 2020. Applying abundance/biomass comparison curves to small mammals: a weak tool for detect urbanizationrelated stress in the assemblages? Folia Oecologica. 47, 10-15. https://doi.org/10.2478/foecol-2020-0002 
Elliott, G., Mitchell, B., Wiltshire, B., Manan, I.A., Wismer, S., 2001. Community participation in marine protected area management Wakatobi National Park, Sulawesi, Indonesia. Coast. Manag. 29, 295-316.https://doi. org/10.1080/089207501750475118

Fanjul, E., Bazterrica, M.C., Escapa, M., Grela, M.A., Iribarne, O., 2011. Impact of crab bioturbation on benthic flux and nitrogen dynamics of Southwest Atlantic intertidal marshes and mudflats. Estuar. Coast. Shelf Sci. 92, 629-638. https://doi.org/10.1016/j.ecss.2011.03.002

Gayanilo, F.C., Sparre, F., Pauly, D., Gayanilo, F.C.Jr., Sparre, P., Pauly, D., 2005. FAO-ICLARM Stock Assessment Tools II (FiSAT II). Revised version. User's guide. FAO, Rome.

Hu, M., Wang, C., Liu, Y., Zhang, X., Jian, S., 2019. Fish species composition, distribution and community structure in the lower reaches of Ganjiang River, Jiangxi, China. Sci. Rep. 9, 1-13. https://doi.org/10.1038/s41598-01946600-2

Hughes, S., Yau, A., Max, L., Petrovic, N., Davenport, F., Marshall, M., McClanahan, T.R., Allison, E.H., Cinner, J.E., 2012. A framework to assess national level vulnerability from the perspective of food security: the case of coral reef fisheries. Environ. Sci. Policy. 23, 95-108. https://doi. org/10.1016/j.envsci.2012.07.012

Lee, S.L., Chong, V.C., Loh, K.H., Yurimoto, T., 2013. Are intertidal mudflat communities (fish and shrimp) affected by cockle culture? Malaysian J. Sci. 32, 107116. https://doi.org/10.22452/mjs.vol32no3.10

Madduppa, H.H., Koropitan, A.F., Damar, A., Subhan, B., Taufik, M, Minsaris, L.O.A., Taurusman, A.A., Ramli, A., Purwanto, A.B., 2020. Ecological vulnerability of coral reef ecosystem in Wakatobi National Park during Indian ocean dipole event. HAYATI J. Biosci. 27, 57-70. https://doi.org/10.4308/hjb.27.1.57

McMellor, S., Smith, D.J., 2013. Coral reefs of the Wakatobi: Abundance and biodiversity. in: Clifton, J., Unsworth, R.K.F., Smith, D.J., (Eds.), Marine Research and Conservation in the Coral Triangle. Operation Wallacea and Gov't of Wakatobi Regency, Wakatobi. pp. 19-33.
Meire, P.M., Dereu, J., 1990. Use of the abundance/biomass comparison method for detecting environmental stress: some considerations based on intertidal macrozoobenthos and bird communities. J. Appl. Ecol. 27, 210-223. https://doi.org/10.2307/2403579

Odum, E.P., 1971. Fundamentals of Ecology, Third ed. Saunders College, Athens.

Pauly, D., 1983. Some simple methods for the assessment of tropical fish stocks. In: FAO. Fisheries Technical Paper. FAO, Rome. pp. 52.

Pet-Soede, C., Van Densen, W.L.T., Pet, J.S., Machiels, M.A.M., 2001. Impact of Indonesian coral reef fisheries on fish community structure and the resultant catch composition. Fish. Res. 51, 35-51. https://doi. org/10.1016/S0165-7836(00)00236-8

Pielou, E.C., 1966. The measurement of diversity in different types of biological collections. J. Theor. Biol. 13, 131144. https://doi.org/10.1016/0022-5193(66)90013-0

Sparre, P., Venema, C.S., 1998. Introduction to tropical fish stock assessment. Part I: Manual. FAO Tech. Pap, Rome.

Wijaya, W., Damanik, J., 2020. Ecotourism study on ecotourism development in Kapota Island, Wakatobi Regency, Southeast Sulawesi Province. E-Journal Tour. 7,300-322. https://doi.org/10.24922/eot.v7i2.63685

Yemane, D., Field, J.G., Leslie, R.W., 2005. Exploring the effects of fishing on fish assemblages using Abundance Biomass Comparison (ABC) curves. ICES Journal of Marine Science. 62, 374-379. https://doi.org/10.1016/j. icesjms.2005.01.009

Yuliana, E., Boer, M., Fahrudin, A., Kamal, M.M., Pardede, S.T., 2019. Using ecosystem approach indicators for assessing the ecological status of reef fisheries management in a marine protected area. Biodiversitas. 20, 1802-1810. https://doi.org/10.13057/biodiv/ d200703

Zheng, Y., Liu, D., 2021. Research on marine pollution problems and solutions in China from the perspective of marine tourism. J. Mar. Sci. 3, 19-28. https://doi. org/10.30564/jms.v3i1.2599 